\title{
Bilateral Spontaneous Achilles Tendon Rupture without Any Predisposing Factors
}

\author{
Esat Uygur ${ }^{1}$, Samet Erinç ${ }^{1}$, Hakan Turan Çift ${ }^{1}$, Feyza Ünlü Özkan ${ }^{2}$, Salih Söylemez ${ }^{1}$ \\ ${ }^{1}$ Trauma and Orthopaedic Surgery Department, İstanbul Medeniyet University Göztepe Research and Training Hospital, İstanbul, \\ Turkey; ${ }^{2}$ Department of Physical Therapy and Rehabilitation, İstanbul Fatih Sultan Mehmet Research and Training Hospital, İstanbul, \\ Turkey. \\ Email: esatuygur@gmail.com
}

Received July $13^{\text {th }}, 2012$; revised August $16^{\text {th }}, 2012$; accepted August $28^{\text {th }}, 2012$

\begin{abstract}
Besides spontaneous rupture of achilles tendon is a rare condition which is usually associated with corticosteroid or fluoroquinolon usage. Spontaneous rupture of achilles tendon without any risk factors is extremely rare. In this report we aimed to present clinical features of a case of bilateral spontaneous achilles tendon rupture and tried to investigate potential etiologic factors. A 54-year-old man was admitted to our emergency department as complaining bilateral ankle pain at the retrocalcaneal region. He had no trauma, any comorbidities or any drug consumption history. Painful achilles tendon gaps proximal to his ankles had been palpated and bilateral Thompson's test positivity had been noted (Figures 1 and 2). He was unable to rise upon his toes however he was able to walk. American Orthopedic Foot and Ankle Society Hindfoot Scoring was calculated as 78 points. The rupture had been verified with a magnetic resonance imaging and his laboratory findings were all within normal limits (Figures 3 and 4). The patient did not approve an operation so conservative treatment with dorsiflexion limiting brace was applied and strengthening and stretching exercises of gastrosoleus was suggested. Atraumatic and bilateral cases with achilles rupture should be well investigated whether any systemic factors are coexistent. Besides well known corticosteroids or fluoroquinolone exposure, endocrinologic and rheumatological diseases, hypercholesterolaemia should be evaluated in terms of predisposing causes. Patients who have to use corticosteroids or fluoroquinolone, should be informed about achilles tendon rupture and be suggested do regular physical exercises in order to reduce the rupture risk. Despite predisposing factors, sometimes nothing can be associated with achilles tendon rupture as in our case. Genetic predisposition or a degenerative process of a tendon due to age may be underlying cause like in rotator cuff tears.
\end{abstract}

Keywords: Spontaneous Achilles Tendon Rupture; Achilles Tendon

\section{Introduction}

It is well known fact that spontaneous rupture of achilles tendon is a rare condition which is associated with corticosteroid or fluoroquinolone usage $[1,2]$. Besides, spontaneous rupture without any risk factors is extremely seldom. In this report we aimed to present clinical features of a case of bilateral spontaneous achilles tendon rupture and emphasize investigating potential etiologic factors.

\section{Case Report}

A 54-year-old man was admitted to our department as complaining bilateral ankle pain at his retrocalcaneal region. In a detailed history it was revealed that he had felt a sudden pain after he experienced a snap at the back of his left ankle as he was descending the stairs a week before. He had been to an orthopedic surgeon whom offered him a surgery due to his achilles tendon rupture.
The patient rejected an operation due to his mild symptoms and preserved walking ability. Subsequent to taking non-steroid and antienflamatuary drugs which had been prescribed by his orthopaedic physician a week ago, he felt an identical snap and pain at his right ankle the day before he came to our hospital, while he was walking on a flat ground. He told no history of trauma, any comorbidities or any drug consumption except for etodolac usage due to mild ankle pain.

An orthopedic examination was performed and existence of achilles tendon gap had been palpated at his both ankles and bilateral Thompson's test positivity had been noted. He was unable to rise upon his toes however his walking ability was preserved with only mild limitation due to limited dorsiflexion .

We applied American Orthopedic Foot and Ankle Society Hindfoot Scoring to the patient and estimated 78 points.

Via the magnetic resonance images we found out the 


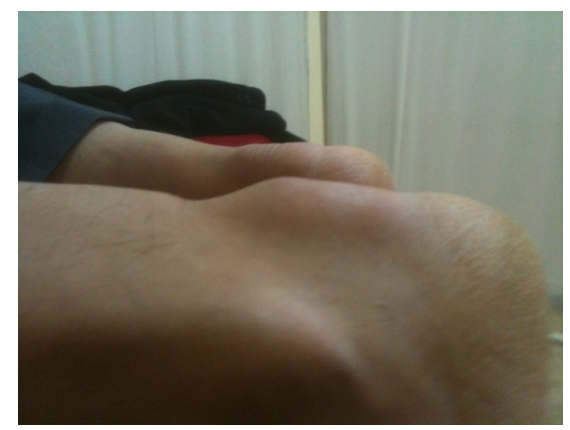

Figure 1. Bilateral gap existence at the achilles tendon.

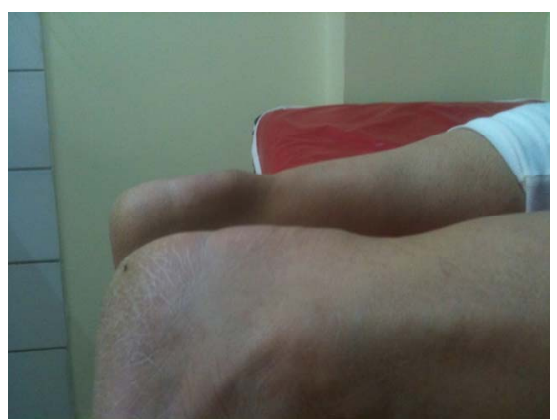

Figure 2. Bilateral gap existence at the achilles tendon.

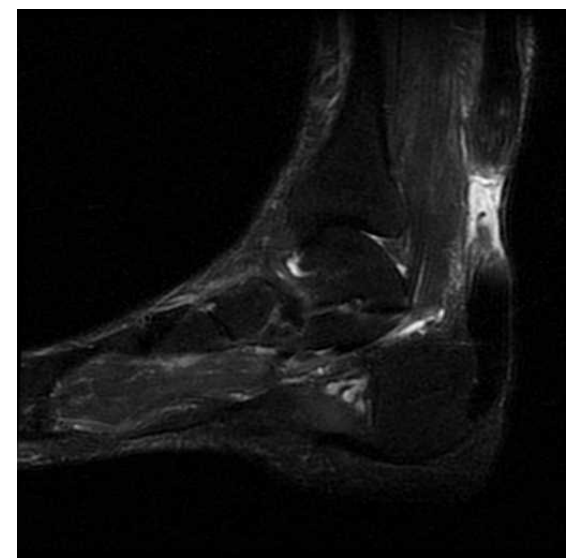

Figure 3. Right ankle achilles tendon rupture.

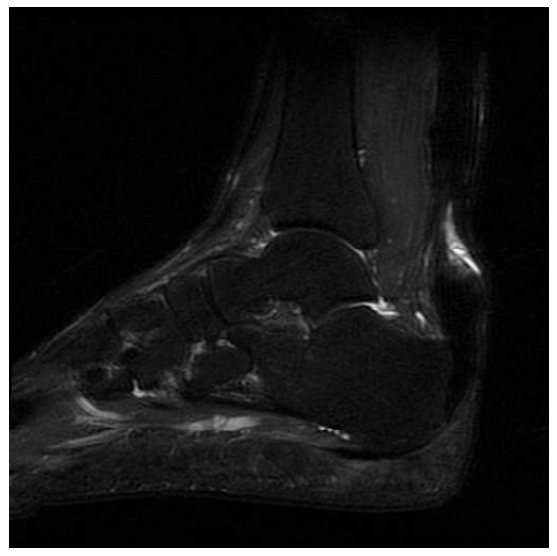

Figure 4. Left ankle achilles tendon rupture. ruptures had been $4 \mathrm{~cm}$ proximal from the calcaneal insertion with $2.5 \mathrm{~cm}$ gaps. Operation was offered but the patient did not approve to be operated so conservative treatment with dorsiflexion limiting brace was applied and strengthening and stretching exercises of gastrosoleus was suggested [3]. Brace was used for 8 weeks and stretching exercises were begun after that.

$2.5 \mathrm{~cm}$ heel lift was added to his shoe and used for subsequent 8 weeks [3]. The patient was seen at his last control 6 months after the rupture with AOFAS score of 95.

\section{Discussion}

Achilles tendon is the most frequently ruptured tendon in the lower extremity and it is usually seen at the active individual men at their fourth or fifth decade of life. Male-female rate is altering between $2 / 1$ and 12/1 [4,5].

At the literature we confront with spontaneous achilles tendon rupture as case reports. Coexistent rheumatological diseases or endocrinopathies, chronical overuse, fluoroquinolone consumption are well defined etiologic factors at spontaneous achilles tendon rupture however the mechanism of underlying tendon degeneration was also described [4]. Besides these factors, Rao et al. reported a 38-year-old woman who had bilateral spontaneous achilles tendon rupture and they proposed that it was associated with hypotyroidism [5]. In some studies correlation with hypercholesterolaemia [6,7], hyperparatyroidism and renal failure $[8,9]$ has been exhibited.

Habusta et al. proposed that chronical overuse in athletes may cause spontaneous achilles tendon rupture [10], they reported two athletes which have been exposed to chronic wear.

Tendinopathy due to fluoroquinolone usage has been firstly demonstrated by Bailey [11]. Following studies indicate that fluoroquinolone type antibiotics lead to structural abnormalities in tenocytes and the presence of giant cells $[12,13]$. Besides achilles tendon rupture, fluoroquinolone utilization may also be associated with patellar and biceps tendonruptures [14]. In some studies it is exhibited that concomitant usage of fluoroquinolone and corticosteroids notably increase the risk of achilles tendon rupture $[1,15]$.

In some cases nothing can be determined as an underlying cause. Hashim et al. [5] and Syed et al. [16] had published their cases without any predisposing risk factors they could find out.

We also investigated the patient's tyroid function tests, hypercholesterolaemia, hyperparatyroidism and judged the patient whether he had used corticosteroids or fluoroquinolone but there had been nothing in order to explain spontaneous rupture.

It is a well known fact that achilles tendon rupture frequently occurs $2-6 \mathrm{~cm}$ proximal of the calcaneal inser- 
tion due to lack of blood supply [4]. It is identical both at traumatic and atraumatic ruptures.

Management of a spontaneous achilles tendon rupture is controversial. Because of an adequate scoring degree (78 points) of American Orthopaedic Foot and Ankle Society score and his refusal to be operated when he applied to our hospital, we had to treat the case conservatively. We had used functional bracing with strengthening and stretching exercises of gastrosoleus muscle. After 6 months of conservative treatment the American Orthopaedic Foot and Ankle Society score of hindfoot mesured as 95 .

Spontaneous rupture of achilles tendon is very rare at the literature. Atraumatic and bilateral cases should be well investigated whether any systemic factors are coexistent. Besides well known corticosteroids or fluoroquinolone exposure, endocrinologic and rheumatismal diseases the patients should be evaluated in terms of predisposing causes such as tyroid function impairment and hypercholesterolaemia. Despite investigating all these factors it should be noted that in some cases nothing could be detected.

In appropriate cases, concomitant utilization of corticosteroids and fluoroquinolones should be avoided as possible.

The patients who have to use corticosteroids or fluoroquinolone should be informed about achilles tendon rupture and be suggested do regular physical exercises in order to reduce the rupture risk. At achilles tendon ruptures we had confronted two different pathologies which are called tendinopathy (tendinitis) and noninflamatuar tendinozis that can lead to spontaneous rupture of Achilles tendon [17]. In an experimental study, Han et al. identified beneficial effects of the extracorporeal shockwave therapy applied to the tendinopathy cases [18]. According to this research, the cases that foresighted to lead tendinopathy should be suggested to regular physical activities. In order to reduce the risk of rupture, the patients should be followed up closely and in a possible tendinopathy situation extracorporeal shockwave therapy should take place as a treatment option. Despite predisposing factors, sometimes nothing can be associated with achilles tendon rupture as in our case. Genetic predisposition or a degenerative process of a tendon due to age may be underlying cause like in rotator cuff tears.

\section{REFERENCES}

[1] C. Parmar and K. P. Meda, "Achilles Tendon Rupture Associated with Combination Therapy of Levofloxacin and Steroid in Four Patients and a Review of the Literature," Foot \& Ankle International, Vol. 28, No. 12, 2007, pp. 1287-1289. doi:10.3113/FAI.2007.1287

[2] Z. Khanzada, U. Rethnam, D. Widdowson and A. Mirza, "Bilateral Spontaneous Non-Traumatic Rupture of the
Achilles Tendon: A Case Report," Journal of Medical Case Reports, Vol. 30, No. 5, p. 263.

[3] G. P. McComis, D. A. Nawoczenski and K. E. DeHaven, "Functional Bracing for Rupture of the Achilles Tendon. Clinical Results and Analysis of Ground-Reaction Forces and Temporal Data," Journal of Bone and Joint Surgery, Vol. 79, No. 12, 1997, pp. 1799-1808.

[4] G. W. Hess, “Achilles Tendon Rupture: A Review of Etiology, Population, Anatomy, Risk Factors, and Injury Prevention," Foot \& Ankle Specialist, Vol. 3, No. 1, 2010, pp. 29-32. doi:10.1177/1938640009355191

[5] Z. Hashim, Z. Dahabreh, M. T. Bin Jemain and H. R. Williams, "Bilateral Simultaneous Achilles Tendon Rupture in the Absence of Risk Factors," Foot \& Ankle Specialist, Vol. 4, No. 3, 2011, pp. 175-178. doi:10.1177/1938640010397657

[6] S. K. Rao, B. C. Navadgi and A. Vasdev, "Bilateral Spontaneous Rupture of Achilles Tendons: A Case Report," Journal of Orthopaedic Surgery, Vol. 13, No. 2, 2011, pp. 178-180.

[7] G. Mathiak, J. V. Wening, M. Mathiak, L. F. Neville and K. Jungbluth, "Serum Cholesterol Is Elevated in Patients with Achilles Tendon Ruptures," Archives of Orthopaedic and Trauma Surgery, Vol. 119, No. 5-6, 1999, pp. 280 284. doi: $10.1007 / \mathrm{s} 004020050410$

[8] N. Basic-Jukic, I. Juric, S. Racki and P. Kes, "Spontaneous Tendon Ruptures in Patients with End-Stage Renal Disease," Kidney and Blood Pressure Research, Vol. 32, No. 1, 2009, pp. 32-36. doi:10.1159/000201792

[9] J. H. Park, S. B. Kim, H. S. Shin, G. H. Jung, Y. S. Jung and H. Rim, "Spontaneous and Serial Rupture of Both Achilles Tendons Associated with Secondary Hyperparathyroidism in a Patient Receiving Long-Term Hemodialysis," International Urology and Nephrology, 2012, in press. doi:10.1007/s11255-011-0111-y

[10] S. F. Habusta, "Bilateral Simultaneous Rupture of the Achilles Tendon. A Rare Traumatic Injury," Clinical Orthopaedics and Related Research, Vol. 320, 1995, pp. 231-234.

[11] R. R. Bailey, J. A. Kirk and B. A Peddie, "NorfloxacinInduced Rheumatic Disease," The New Zealand Medical Journal, Vol. 27, No. 96, 1983, p. 590.

[12] J. M. Casparian, M. Luchi, R. E. Moffat and D. Hinthorn, "Quinolones and Tendon Ruptures," Southern Medical Journal, Vol. 93, No. 5, 2000, pp. 488-491.

[13] R. M. Harrell, "Fluoroquinolone-Induced Tendinopathy: What Do We Know?" Southern Medical Journal, Vol. 92, No. 6, 1999, pp. 622-625. doi:10.1097/00007611-199906000-00014

[14] D. J. Stinner, J. D. Orr and J. R. Hsu, "FluoroquinoloneAssociated Bilateral Patellar Tendon Rupture: A Case Report and Review of the Literature," Military Medicine, Vol. 175, No. 6, 2010, pp. 457-459.

[15] P. D. Van der Linden, M. C. Sturkenboom, R. M. Herings, H. M. Leufkens, S. S. Rowlands and B. H. Stricker, "Increased Risk of Achilles Tendon Rupture with Quinolone Antibacterial Use, Especially in Elderly Patients Taking Oral Corticosteroids," Archive of Internal Medicine, Vol. 
163, No. 15, 2003, pp. 1801-1807. doi:10.1001/archinte.163.15.1801

[16] S. Syed, A. Bhatti and M. M. Shah, "Spontaneous Atraumatic Achilles Tendon Rupture in Healthy Individuals: Biomechanical Aspect," Journal of Journal of College of Physicians and Surgeons Pakistan, Vol. 19, No. 3, 2009, pp. 195-197.

[17] E. Bass, "Tendinopathy: Why the Difference between Tendinitis and Tendinosis Matters," International Journal of Therapeutic Massage and Bodywork, Vol. 5, No. 1, 2012, pp. 14-17.

[18] S. H. Han, J. W. Lee, G. P. Guyton, B. G. Parks, J. P. Courneya, L. C. Schon and J. Leonard, "Goldner Award 2008. Effect of Extracorporeal Shock Wave Therapy on Cultured Tenocytes," Foot \& Ankle International, Vol. 30, No. 2, 2009, pp. 93-98. doi:10.3113/FAI.2009.0093 